\title{
Resiliencia, vulnerabilidad y sustentabilidad de sistemas socioecológicos en México
}

\author{
Resilience, vulnerability and sustainability of socioecological systems in Mexico \\ Patricia Balvanera $^{\mathrm{a}, *}$, Marta Astier ${ }^{\mathrm{b}}$, Francisco D. Gurri ${ }^{\mathrm{c}}$ e Isela Zermeño-Hernández ${ }^{\mathrm{a}}$ \\ ${ }^{a}$ Instituto de Investigaciones en Ecosistemas y Sustentabilidad, Universidad Nacional Autónoma de México, Antigua Carretera a Pátzcuaro Núm. 8701, Col. \\ Ex-Hacienda de San José de la Huerta, 58190, Morelia, Michoacán, México \\ ${ }^{\mathrm{b}}$ Instituto de Investigaciones en Geografía Ambiental, Universidad Nacional Autónoma de México, Antigua Carretera a Pátzcuaro Núm. 8701, Col. Ex-Hacienda \\ de San José de la Huerta, 58190, Morelia, Michoacán, México \\ ${ }^{\mathrm{c}}$ Departamento Ciencias de la Sustentabilidad, El Colegio de la Frontera Sur-Unidad Campeche, Av. Rancho Polígono 2-A, Col. Ciudad Industrial, 24500 Lerma \\ Campeche, Campeche, México \\ Recibido el 10 de junio de 2016; aceptado el 13 de septiembre de 2017 \\ Disponible en Internet el 28 de noviembre de 2017
}

\section{Resumen}

Existe un creciente entendimiento de una fuerte interdependencia entre las sociedades y los ecosistemas, y de esta visión surge como parte de un nuevo paradigma el concepto de sistema socioecológico (SS). En este artículo se presenta un análisis de las contribuciones de los académicos mexicanos al análisis de los SS. Primero, revisamos los conceptos básicos de sistemas socioecológicos, resiliencia, vulnerabilidad y servicios ecosistémicos en el contexto de los SS. Segundo, analizamos las aportaciones teórico-conceptuales de los científicos mexicanos a estos temas. Tercero, describimos algunos estudios de caso sobresalientes sobre el análisis integral de SS. Cuarto, exploramos cuantitativamente el aporte de los académicos mexicanos en publicaciones sobre SS, resiliencia y sustentabilidad. Quinto, analizamos los programas educativos de licenciatura y posgrado sobre estos temas en México. Sexto, describimos cómo estos enfoques han permeado en la toma de decisiones. Finalmente, hacemos un análisis de los vacíos del conocimiento, retos y perspectivas, identificando los avances importantes en el tema de SS, resiliencia y vulnerabilidad en el país. Consideramos fundamental entender los retos relacionados con la estabilidad de los sistemas, la certidumbre científica y la existencia de expertos tomadores de decisiones así como el desarrollo de SS más resilientes y menos vulnerables.

(C) 2017 Universidad Nacional Autónoma de México, Instituto de Biología. Este es un artículo Open Access bajo la licencia CC BY-NC-ND (http://creativecommons.org/licenses/by-nc-nd/4.0/).

Palabras clave: Exposición; Sensibilidad; Servicios ecosistémicos; Capacidad adaptativa; Adaptabilidad; Sustentabilidad; Licenciatura; Posgrado; Desastres

\section{Abstract}

There is a growing understanding of the strong interdependence between societies and ecosystems, and from this vision the concept of socialecological system (SS) arises, as part of a new paradigm. This article presents an analysis of the contributions of Mexican scholars to the analysis of SS. First, we review the basic concepts of socioecological systems, resilience, vulnerability and ecosystem services in the context of SS. Second, we analyze the theoretical-conceptual contributions of Mexican scientists to these issues. Third, we describe some outstanding case studies of the SS integrative perspective. Fourth, we quantitatively explore the contribution of Mexican scholars in publications on SS, resilience and sustainability.

\footnotetext{
* Autor para correspondencia.

Correo electrónico: pbalvanera@cieco.unam.mx (P. Balvanera).
} 
Fifth, we assess the undergraduate and postgraduate educational programs on these topics in Mexico. Sixth, we describe how these approaches have permeated decision-making. Finally, we analyze knowledge gaps, challenges and perspectives, identifying the important advances in SS, resilience and vulnerability in the country. We consider critical to understand the challenges related to the stability of the systems, the scientific certainty and the existence of expert decision makers as well as the development of more resilient and less vulnerable SS.

(C) 2017 Universidad Nacional Autónoma de México, Instituto de Biología. This is an open access article under the CC BY-NC-ND license (http://creativecommons.org/licenses/by-nc-nd/4.0/).

Keywords: Exposure; Sensitivity; Ecosystem services; Adaptive capacity; Adaptability; Sustainability; Bachelor's degree; Postgraduate; Disasters

\section{Introducción}

\section{Contexto del tema en México y en el mundo}

En la cosmovisión de los habitantes del mundo prehispánico no existía una dicotomía entre el ser humano y el ambiente. Las tragedias podían ser consecuencia de una interacción, ya sea material o espiritual, inadecuadas, o lo que ahora denominamos un mal manejo. Desafortunadamente, otras visiones, como las que dominan en el mundo occidental, solo han aceptado que las sociedades son parte de la naturaleza y no dueñas de esta a raíz del grave deterioro ambiental que vivimos ahora.

El concepto de «sistema socioecológico» nace, en un mundo de científicos que diferencian entre el ser humano y el mundo que lo rodea, como parte de un nuevo paradigma que entiende que la actividad humana existe en un sistema de interacciones complejas de interdependencia entre los distintos componentes sociales y ecológicos (Binder, Hinkel, Bots y Pahl-Wostl, 2013; Fischer et al., 2015). Esta visión revolucionaria la integran científicos con distintas formaciones y marcos epistémicos que borran la frontera tradicional entre las ciencias sociales y naturales en su preocupación por la conservación de la biodiversidad y por asegurar el adecuado funcionamiento de los ecosistemas y el de los sistema de soporte de la vida en el planeta (Liu et al., 2007). Surge así el interés por entender cómo las sociedades coevolucionan junto con el ambiente que las rodea, cómo las relaciones de poder median las interacciones ambiente-sociedad, los usos que hacen las sociedades de los componentes de los ecosistemas, así como el análisis del metabolismo social que hace una analogía entre ecosistemas y sociedades para visibilizar las contribuciones de los ecosistemas a satisfacer las necesidades de las interacciones entre sociedades y su entorno en el espacio y el tiempo (Balvanera et al., 2011; Brondízio y Moran, 2012).

El análisis de los sistemas socioecológicos utiliza un conjunto de acercamientos que se conocen como las Ciencias de la Sustentabilidad. En este manuscrito hacemos un esfuerzo por integrar el concepto de vulnerabilidad desarrollado en las ciencias sociales con el de resiliencia utilizado en las ciencias ambientales y, de esta manera, generar un lenguaje común que facilite los esfuerzos multidisciplinarios. Originalmente el concepto de vulnerabilidad surge de la necesidad de lidiar con las tragedias humanas y económicas generadas por desastres naturales o aquellos provocados por el ser humano. La vulnerabilidad puede descomponerse en: exposición, sensibilidad y resiliencia. El primero se refiere a eventos potencialmente catastróficos que tienen una frecuencia, duración y magnitud y que suceden en un contexto biogeofísico dado (Ahn y Choi, 2013; Messner y
Meyer, 2006; Soares, Murillo, Romero y Millán, 2014; Tucker, Eakin y Castellanos, 2010; Wong y Zhao, 2001) y en una población con una capacidad de mitigación definida (López-Marrero, 2010; Messner y Meyer, 2006; Tucker et al., 2010; VergaraTenorio et al., 2011). La sensibilidad, mide cómo las condiciones socioecológicas existentes les permiten a los individuos o a las comunidades absorber cambios generados por fenómenos catastróficos (Adger, 2006; Burton y Lim, 2001; Gallopín, 2006; O’Brien, Sygna y Haugen, 2004; Smit y Pilifosova, 2001). La sensibilidad se acerca a lo que se conoce como vulnerabilidad social y, puesto que puede variar independientemente de la existencia del fenómeno de riesgo, puede conceptualizarse como un elemento único de la vulnerabilidad. Finalmente, la resiliencia se refiere a la capacidad del sistema para absorber perturbaciones y mantener sus funciones, así como la de renovarse y reorganizarse. La resiliencia depende tanto de las condiciones de los recursos naturales (suelo, agua, y biodiversidad) como del nivel de conocimiento y de la capacidad de aprender y de gestionar de los grupos humanos y de sus instituciones (Astier, Speelman, López-Ridaura, Masera y González-Esquivel, 2011; Berkes, Folke y Colding, 2000; Berkes y Jolly, 2002).

El concepto de «servicios ecosistémicos» surge como precursor de las visiones socioecológicas para hacer patente la interdependencia entre las sociedades y la naturaleza (Daily, 1997). Se concibió originalmente los servicios ecosistémicos, o beneficios que obtenemos de los ecosistemas, como un flujo direccional de los ecosistemas a la sociedad, abarcando el flujo de bienes tangibles, la regulación de las condiciones en las que las sociedades viven y realizan sus actividades productivas, así como beneficios no tangibles que surgen como experiencias o capacidades (Chan et al., 2012; MA, 2005; Maass et al., 2005). Actualmente se discute que son más bien las interacciones entre las sociedades y la naturaleza las que dan de forma conjunta el surgimiento de beneficios para la sociedad (Palomo, FelipeLucía, Bennett, Martín-López y Pascual, 2016).

El concepto de resiliencia surge del estudio empírico de los sistemas que nos rodean, desde la observación de fenómenos que operan a múltiples escalas espaciales y temporales, como podría ser el caso de fenómenos ambientales como las sequías y sus consecuencias. Sin embargo, puesto que la recuperación de su función puede ser distinta a la forma, y cambios en los sistemas socioecológicos pueden ser modificados por su propia dinámica, lo que se conoce como adaptabilidad (García-Barrios, Speelman y Pimm, 2008), la recuperación no solo puede tardar una cantidad de tiempo indeterminada, sino que será difícil de detectar. En el estudio de posibles catástrofes surge el concepto de «capacidad adaptativa» como la habilidad del sistema 
de reducir los impactos negativos de una catástrofe y tomar ventaja de oportunidades para recuperarse (Nelson, Adger y Brown, 2007; Smit y Pilifosova, 2001). La capacidad adaptativa (o adaptabilidad) se centra en la capacidad que poseen los afectados para tomar las decisiones que llevan a procesos de gobernanza y autogestión además de poder crear instituciones y política pública. Se trata de promover estrategias que contribuyan a generar adaptaciones sustentables, realistas en el contexto de las restricciones propias del sistema, a los cambios estructurales que sufre el sistema socioecológico (Eakin y Luers, 2006).

\section{Aportaciones teórico-conceptuales por autores mexicanos}

En México diferentes tradiciones abrieron barreras disciplinarias para permitir la conceptualización de los elementos del sistema socioecológico como una herramienta teórica para promover la sustentabilidad. Estas son la etnoecología (e.g., Caballero et al., 1978), la ecología cultural (Palerm, 1989), la ecología política (Durand-Smith, 2012; Durand-Smith, Figueroa-Díaz, Chávez y Genet, 2011) y la ecología humana (Daltabuit, Licón, Lozano y Ricco, 1988). Surgen así la incorporación de la sustentabilidad desde la ecología de ecosistemas (Maass, 2012), el análisis sobre el concepto de interdisciplina y sistemas complejos socioambientales (García, 2006), la filosofía multidisciplinaria del programa Hombre en la Biosfera (MAB) y la esperanza de desarrollar mecanismos financieros para asegurar el mantenimiento de la biodiversidad a través de compensaciones económicas por los servicios brindados (e.g., REDD+; Torres-Rojo y Sanginés, 2002). Desafortunadamente, y aunque existe comunicación entre científicos de las distintas disciplinas, este esfuerzo multidisciplinario hacia la sustentabilidad se ha desarrollado de manera casi independiente entre distintos grupos multidisciplinarios que han generado homónimos con significados distintos aunque no del todo incompatibles.

Varios conjuntos de investigadores de distintas disciplinas se agruparon en redes inter- y multidisciplinarias, lo que permitió avanzar en el desarrollo del concepto de sistemas socioecológicos. Estas incluyen a la Red Mexicana de Investigación Ecológica de Largo Plazo (Jardel, Maass y Rivera-Monroy, 2013), la Red de Estudios Sociales en Prevención de Desastres en América Latina (http://www.la-red.org) y el Grupo de Investigación MESMIS (http://mesmis.gira.org.mx). La primera, agrupa a biólogos y ecólogos interesados en la conservación de sistemas naturales y enfatiza la necesidad de un abordaje sistémico más allá de estos, abarcando las complejas interacciones entre este, las sociedades y su manejo (Maass, Díaz-Delgado, Balvanera, Castillo y Martínez-Yrízar, 2010). La participación de varios autores mexicanos en la Evaluación de los Ecosistemas del Milenio (MA, 2005), así como la elaboración del libro Capital Natural de México (Sarukhán y Soberón, 2008) siguiendo un acercamiento similar, fortalecen el acercamiento de sistema socioecológico. De ahí mismo surge el énfasis en el concepto de «servicios ecosistémicos», el cual es promovido por su utilidad para entender las interacciones que ocurren al interior de los sistemas socioecológicos (Balvanera y Cotler, 2011). Surgen también mecanismos financieros para asegurar el mantenimiento de la biodiversidad a través de compensaciones económicas por los servicios ecosistémicos brindados (TorresRojo y Sanginés, 2002).

Los primeros grupos de investigación en el país interesados en poner en práctica el concepto de resiliencia, en el contexto de sustentabilidad de los sistemas agropecuarios, incluyen a la Red Mex-LTER y al Grupo de Investigación MESMIS. El primero ha impulsado tanto a nivel nacional (Maass et al., 2010) como internacional (Maass y Equihua, 2015) el tránsito de la investigación ecológica de largo plazo (LTER) a la investigación socioecosistémica de largo plazo (LTSER). El Grupo MESMIS se crea para estudiar el desempeño, en términos de sustentabilidad, de los sistemas de manejo de recursos naturales alternativos en México (Masera, Astier y López-Ridaura, 1999). Este genera un marco metodológico para evaluar diferentes propuestas de manejo de sistemas agrícolas, forestales, agroforestales, en contextos geográficos muy diferentes, que se aplica fundamentalmente a pequeños agricultores en comunidades campesinas (López-Ridaura, Masera y Astier, 2002). El marco identifica variables críticas de desempeño de los sistemas (e.g., la productividad para un agroecosistema), así como mecanismos internos que aseguren el mantenimiento de estas variables en un rango adecuado a pesar de cambios sociales y ambientales. Se generan así predictores de la resiliencia, incluyendo indicadores de la biodiversidad, en los agroecosistemas (Aguilar-Støen, Moe y Camargo-Ricalde, 2009). Se exploran las variables asociadas a la resiliencia y adaptabilidad para una abanico de estudios de caso en México (Astier, García-Barrios, Galván-Miyoshi, González-Esquivel y Masera, 2012), así como las características que determinan la resiliencia de hogares y sistemas de maíz campesino después de un año de sequía en la Región Purhépecha (Arnés, Antonio, del Val y Astier, 2013).

La sustentabilidad de sistemas socioecológicos urbanos y rurales encuentra su expresión más incluyente en el estudio de vulnerabilidad ante desastres potenciales. La Red de Estudios Sociales en Prevención de Desastres en América Latina (GarcíaAcosta, 2008) genera el concepto de «Gestión de Riesgo». A 25 años de su fundación autores mexicanos y latinoamericanos han contribuido a plasmar la idea de que los desastres no suceden sino que se forjan como parte de la historia de los pueblos que la sufren. La vulnerabilidad, y por supuesto la exposición, sensibilidad y resiliencia que la componen, son parte de la sustentabilidad que se construye históricamente. La vulnerabilidad puede ser medida y el conocimiento de sus características puede contribuir a mitigar, y aumentar la adaptabilidad y resiliencia de los sistemas socioecológicos. El conocimiento de lo que genera el desastre ha permitido el desarrollo en México de una serie de índices que incluyen el índice de vulnerabilidad social y su distribución espacial. Este es el caso de las entidades federativas de México, 1990-2010 elaborado por Reyna Vergara González de la Universidad Autónoma del Estado de México y el índice de vulnerabilidad social municipal, México (Conapo, 2013) entre otros.

\section{Estudios de caso sobresalientes del tema en México}

Probablemente el estudio de caso más icónico para el análisis integral del sistema socioecológico y los servicios ecosistémicos 
es aquel relacionado con los servicios que brinda el bosque tropical seco en la Reserva de la Biosfera Chamela-Cuixmala y sus alrededores. En 1980 se establecen 5 cuencas experimentales para el estudio del funcionamiento del ecosistema: estructura y dinámica de la vegetación, ciclos hidrológicos y ciclos biogeoquímicos (Maass, Martínez-Yrízar, Patiño y Sarukhán, 2002; Sarukhán y Maass, 1990). Se analiza también la forma en la que el bosque tropical seco es transformado en campos agrícolas y pecuarios. En este sitio convergen científicos naturales con distintas perspectivas y formaciones y se forma ahí la semilla de la Red Mex-LTER. A partir de finales de los 90 se unen al equipo científicos sociales que analizan la conservación desde la perspectiva de los pobladores locales. A través de equipo interdisciplinarios cada vez más diversos e integrados, se genera una síntesis sobre los servicios ecosistémicos que este sistema socioecológico ofrece (Balvanera et al., 2011; Maass et al., 2005), se analiza todo el sistema socioecológico de la cuenca del río Cuixmala (Balvanera et al., 2011; Castillo, 2011), así como la vulnerabilidad del sistema socioecológico al cambio global (Balvanera et al., 2011; Gavito et al., 2014; Maass et al., 2005). Actualmente se encuentran en proceso proyectos para evaluar la respuesta del socioecosistema a los huracanes, la resiliencia del sistema de producción pecuaria, la recuperación de biodiversidad, funciones y servicios en los bosques secundarios, así como el papel de la biodiversidad en la mitigación del cambio climático.

Uno de los sistemas de estudio mejor analizado en términos de su resiliencia y adaptabilidad con la metodología del grupo MESMIS lo forman los sistemas de manejo campesinos en la Región Purhépecha, México (Arnés et al., 2013). Dichas comunidades son representativas en cuanto a la práctica de un sistema agropecuario y el uso de variedades nativas de cultivos y maíz en el marco de la milpa y la diversificación en el uso del paisaje. Se han desarrollado, de forma participativa, indicadores sociales, económicos y ecológicos para analizar la resiliencia, adaptabilidad y productividad de los sistemas evaluados. Se ha analizado el papel de la sequía a través de medidas durante más de 3 años consecutivos en la comunidad de Napízaro, en donde se presentó una sequía en el 2011. Los resultados obtenidos en colaboración con 12 ejidatarios reflejan que los sistemas con un reducido uso de insumo, basado en los fertilizantes orgánicos y la rotación, mostraron desempeños comparables con aquellos con un uso intensivo de agroquímicos. En el año en el que se presentó la sequía junto con heladas tempranas la productividad se redujo dramáticamente, pero fueron los sistemas diversificados los que resistieron mejor las adversidades climáticas (Arnés et al., 2013).

Existen pocos estudios longitudinales a nivel mundial que puedan observar el nivel de vulnerabilidad de una población, antes y después de un evento y, de esta manera, medir su resiliencia. En México, el equipo de Antropología Ambiental y Género de El Colegio de la Frontera Sur (Vallejo-Nieto, Gurri-García y Molina-Rosales, 2011) tuvo la oportunidad de analizar las decisiones que se tomaban en el contexto de unidades domésticas campesinas, en el municipio de Calakmul, Campeche, antes de estar expuestas y a lo largo del huracán Isidore, en el año 2001. Dicho estudio se prolongó y se pudo analizar también cómo dichas unidades afrontaron la sequía que se presentó después del huracán. Según el objetivo del sistema agrícola y familiar, se identificaron 2 grupos diferenciados: los que practicaban la agricultura como negocio y los que la practicaban como una estrategia más de subsistencia. Los primeros incurrieron en pérdidas al invertir en rescatar su monocultivo comercial y animales domésticos para poder participar en el mercado ese año o el siguiente por lo que vieron mermados sus ahorros. Los segundos abandonaron un cultivo comercial no redituable y se comieron los animales que la sequía no les permitía mantener. Rescataron los cultivos orientados a cubrir sus necesidades inmediatas y aceleraron su siembra de milpa de ciclo corto, obteniendo mejores rendimientos que el año anterior al huracán por lo que su capacidad de reempezar el ciclo agrícola no se vio mermada. Aunque ambas estrategias son difíciles de comparar en términos comerciales, la que hace agricultura como negocio resultó ser claramente más vulnerable.

\section{Publicaciones sobre el tema por académicos en instituciones mexicanas}

A partir del año 2000 se da un incremento exponencial en el número de productos publicados por académicos mexicanos tanto en inglés como en español sobre sistemas socioecológicos, resiliencia y vulnerabilidad (fig. 1; apéndices 1, 2).
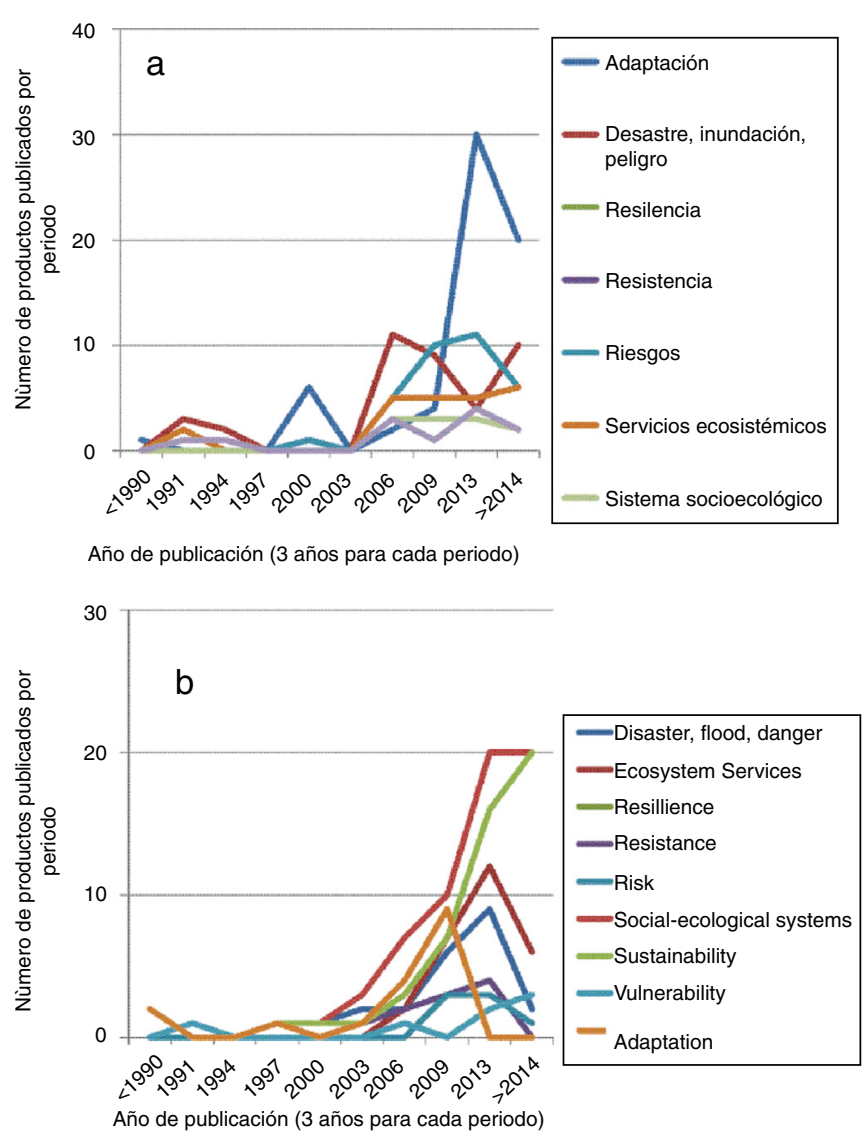

Figura 1. Número de publicaciones sobre sistemas socioecológicos, resiliencia y vulnerabilidad por autores mexicanos por palabra clave. En español (a), en inglés (b). 

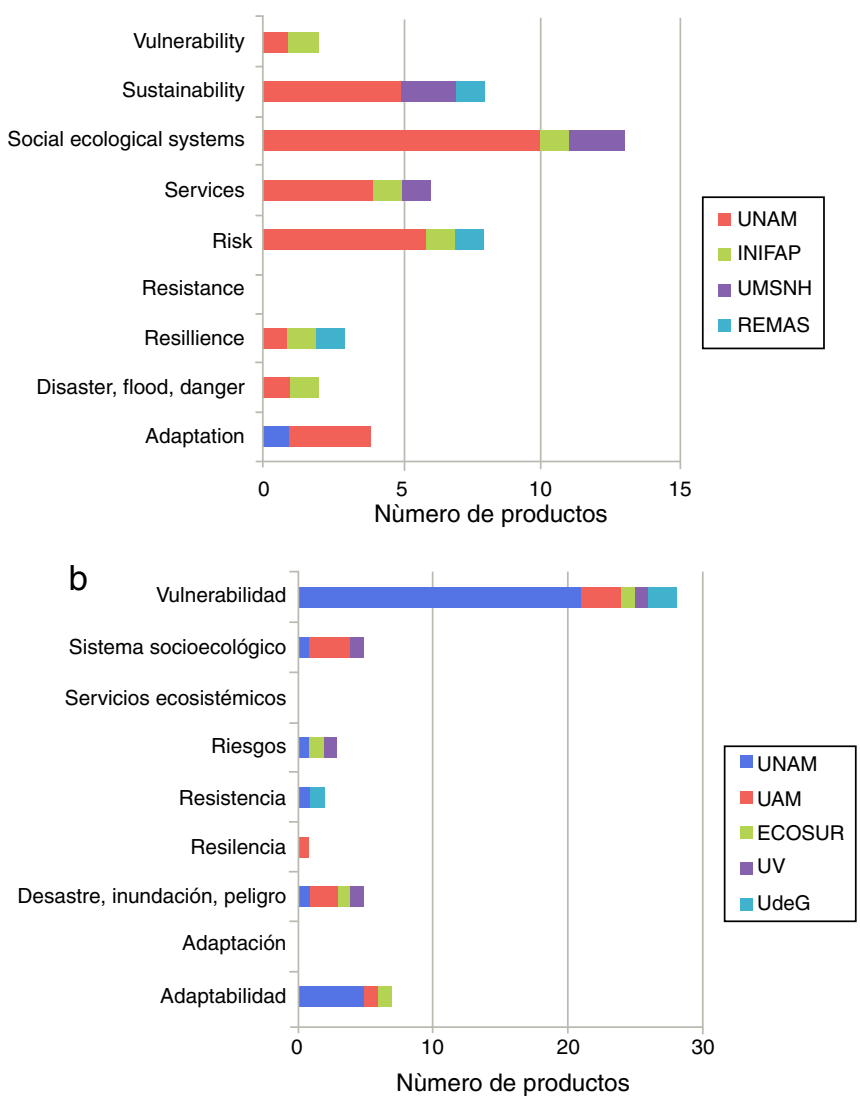

Figura 2. Instituciones mexicanas que contribuyen con el mayor número de publicaciones sobre sistemas socioecológicos, resiliencia y vulnerabilidad para productos (en inglés, a; en español, b).

Destacan en particular las publicaciones en inglés sobre sistemas socioecológicos, resiliencia, y en menor medida sobre servicios ecosistémicos, adaptación y desastres (incluyendo inundaciones y peligros), así como las publicaciones en español sobre vulnerabilidad.

Las instituciones mexicanas que destacan en cuanto a su contribución a los temas de sistemas socioecológicos, resiliencia y vulnerabilidad en México incluyen en primer lugar las distintas dependencias de la Universidad Nacional Autónoma de México (fig. 2, apéndices 1, 2). La Universidad Autónoma Metropolitana, El Colegio de la Frontera Sur, la Universidad Veracruzana, la Universidad Autónoma de Veracruz, el Instituto Nacional de Investigaciones Forestales, Agrícolas y Pecuarias, la Universidad Michoacana de San Nicolás de Hidalgo, el Centro de Investigaciones y Estudios Superiores en Antropología Social y la Red Temática de Medio Ambiente y Sustentabilidad (ReMaS) destacan entre aquellas que pudieron ser identificadas por la búsqueda sistemática que realizamos

\section{Entidades académicas y programas educativos de} licenciatura y posgrado en México que enseñan y/o forman recursos humanos en el campo de estudio correspondiente

En México, un total de 84 programas de estudios en diferentes instituciones mexicanas cuentan con temas de interés potencial para la formación de profesionistas dedicados al estudio de sistemas socioecológicos, resiliencia, vulnerabilidad y sustentabilidad (tabla 1, apéndice 3). De los programas de estudio identificados en 27 estados del país, 3 son especialidades, 68 maestrías y 13 doctorados. Dentro de su plan de estudios se imparten cursos relacionados con los temas de sustentabilidad, ecosistemas, cambio climático, conservación, energía, política y legislación ambiental, entre otros.

Programas educativos como la Maestría en Ciencias en Estudios Ambientales y de la Sustentabilidad del Instituto Politécnico Nacional, la Maestría en Gestión Ambiental para la Sustentabilidad de la Universidad Veracruzana, el Doctorado interinstitucional en Ciencias en Ecología y Desarrollo Sustentable del ECOSUR y el Doctorado en Ciencias de la Sostenibilidad de la UNAM, abordan el área de la sustentabilidad de manera directa y como marco principal y ofrecen una gran cantidad de cursos relacionados directamente con esta temática. Por ejemplo, los Posgrados en Ciencias Biológicas y de la Sostenibilidad, integran las ciencias naturales y sociales, ingeniería y urbanismo. Con esto se pretende generar propuestas innovadoras para formar profesionales que contribuyan al desarrollo sostenible del país. El Posgrado en Ciencias de la Sostenibilidad pretende formar a expertos y científicos que dominen las bases conceptuales y metodológicas de las ciencias de la sostenibilidad, y que sean capaces de proponer soluciones, desde una perspectiva transdisciplinaria.

Otros programas están dirigidos a la conservación de recursos naturales, como la Maestría en Ciencias en Conservación y Aprovechamiento de Recursos Naturales del Instituto Politécnico Nacional o la Maestría en Ciencias en Manejo de Recursos Naturales de la Universidad de Guadalajara. Hay diversos programas dirigidos a la Educación Ambiental que cuentan con ciertos cursos relacionados con el desarrollo sustentable como son: la Maestría en Ciencias Ambientales de la Universidad Autónoma de San Luis Potosí o la Maestría en Ciencias y Doctorado en Ciencias Ambientales de la Benemérita Universidad Autónoma de Puebla.

Con respecto a los cursos intensivos y diplomados relacionados con sistemas socioecológicos, resiliencia y vulnerabilidad se encontraron un total de 77 cursos intensivos con una duración que va desde 4 h hasta 6 meses. De estos cursos 61 diplomados, 15 cursos y un taller son impartidos en 17 estados de la República Mexicana. Además se ofertan actualmente en línea 10 cursos internacionales tipo MOOC (acrónimo en inglés de Massive Open Online Course). Cabe destacar el programa LEAD, auspiciado por El Colegio de México, por el cual han pasado 22 generaciones de profesionistas que hoy se han integrado a distintos ámbitos laborales incluyendo la investigación, la gestión gubernamental, el sector empresarial, con una visión de sostenibilidad.

La mayoría de los cursos intensivos registrados son diplomados y cursos enfocados al desarrollo sustentable, a la gestión y educación ambiental, a la política y gobernanza de los recursos naturales o a la conservación de recursos y a la normatividad ambiental que en su objetivo tienen establecido un interés en la sustentabilidad ambiental. Estos cursos y diplomados son impartidos tanto presencialmente como en línea, con costo o 
Tabla 1

Posgrados y licenciaturas en México que incluyen los sistemas socioecológicos, la resiliencia o la vulnerabilidad en sus planes de estudios.

\begin{tabular}{|c|c|c|}
\hline Universidad & Programa & Link (Plan de estudios) \\
\hline $\begin{array}{l}\text { Universidad Nacional de Estudios } \\
\text { Superiores Unidad Morelia }\end{array}$ & $\begin{array}{l}\text { Licenciado en Estudios Sociales } \\
\text { y Gestión Local }\end{array}$ & $\begin{array}{l}\text { http://oferta.unam.mx/carrera/archivos/planes/estudiossocialesygestionlocal- } \\
\text { enesmorelia-plan-estudios.pdf }\end{array}$ \\
\hline Universidad Nacional de Estudios & Licenciado en Ciencias & https://escolar1.unam.mx/planes/morelia/tec_manejo_informacion.pdf \\
\hline Superiores Unidad Morelia & Ambientales & \\
\hline Flacso México & $\begin{array}{l}\text { Maestría en Población y } \\
\text { Desarrollo }\end{array}$ & http://www.flacso.edu.mx/posgrados/programas_presenciales/mpyd \\
\hline UNAM & $\begin{array}{l}\text { Maestría en Ciencias de la } \\
\text { Sostenibilidad }\end{array}$ & http://www.posgrado.unam.mx/sostenibilidad/pcs-maestr\%C3\%ADa.html \\
\hline UNAM & $\begin{array}{l}\text { Doctorado en Ciencias de la } \\
\text { Sostenibilidad }\end{array}$ & http://www.posgrado.unam.mx/sostenibilidad/pcs-doctorado.html \\
\hline CIAD & $\begin{array}{l}\text { Doctorado en Desarrollo } \\
\text { Regional (DDR) }\end{array}$ & $\begin{array}{l}\text { http://www.ciad.mx/posgrado/122-posgrado/892-doctorado-en-desarrollo- } \\
\text { regional.html }\end{array}$ \\
\hline UNAM & $\begin{array}{l}\text { Doctorado en Geografía } \\
\text { Ambiental }\end{array}$ & $\begin{array}{l}\text { http://www.igeograf.unam.mx/sigg/utilidades/docs/pdfs/posgrados/Ade_y_Mod_ } \\
\text { Mae_Geo_y_Doc_Geo_Tomo_I_11enero2013-1.pdf }\end{array}$ \\
\hline Universidad de Guadalajara & $\begin{array}{l}\text { Maestría en Gestión y Desarrollo } \\
\text { Social }\end{array}$ & $\begin{array}{l}\text { http://www.udg.mx/es/oferta-academica/posgrados/maestrias/maestria-en-gestion- } \\
\text { y-desarrollo-social-cucsh }\end{array}$ \\
\hline UABCS & $\begin{array}{l}\text { Posgrado en Ciencias Marinas y } \\
\text { Costeras }\end{array}$ & $\begin{array}{l}\text { http://www.uabcs.mx/files/cimacoDoctorado//B)\%20ESTUDIANTES//CRITERIO } \\
\% 205 . \% 20 \mathrm{MOVILDAD} \% 20 \mathrm{DE} \% 20 \text { ESTUDIANTES/5.3\%20Proyectos } \% 20 \\
\text { terminales } \% 20 \mathrm{o} \% 20 \text { tesis } \% 20 \text { codirigidas.pdf }\end{array}$ \\
\hline
\end{tabular}

gratuitos y dirigidos a profesionales, investigadores, tomadores de decisiones y estudiantes.

\section{Aportes a la aplicación del conocimiento}

La visión de sistemas socioecológicos y de servicios ecosistémicos está permeando en la toma de decisiones en México. Estos enfoques permiten tener una visión sistémica del país y la generación de políticas públicas transversales, como lo muestra para estudios de caso concretos el libro de Galán, Balvanera y Castellarini (2014). Asimismo, los trabajos de Challenger exploran cómo el concepto de sistemas socioecológicos se está operacionalizando en la gestión ambiental de México (Challenger, 2016; Challenger, Bocco, Equihua, Chavero y Maass, 2014). A la fecha se están explorando las implicaciones del enfoque socioecológico en la salud, el diseño urbano y el diseño de instituciones para la toma de decisiones entre otros temas. El concepto de servicios ecosistémicos, fundamentalmente por su asociación con estímulos financieros a través de pagos que favorecen la conservación de la cobertura vegetal, ha tenido un profundo impacto en México.

Los conceptos de resiliencia y adaptabilidad han sido de gran importancia para su aplicación al diseño de alternativas de manejo agropecuario. Los sistemas socioecológicos agrosilvopastoriles podrán ser resilientes a eventualidades de tipo climático y económico si conservan sus recursos naturales base, además de una agrobiodiversidad funcional (Arnés et al., 2013). Las condiciones climatológicas en el país para el cultivo de maíz están suponiendo grandes desafíos a los sistemas hoy en día y serán todavía más restrictivas en el futuro, por lo que es urgente la aplicación de medidas de adaptación (Monterroso-Rivas, Conde-Álvarez, Rosales-Dorantes, Gómez-Díaz y Gay-García, 2011). Existe un conocimiento y prácticas de manejo de los recursos que han permitido históricamente que los sistemas campesinos hayan sido resilientes a sequías, heladas, granizo, inundaciones, caída de precios e incrementos de precios de los insumos (Arnés et al., 2013; Conde, Ferrer y Orozco, 2006; Rogé y Astier, 2015). Hay esfuerzos aislados a lo largo de todo el país, en su mayoría promovidos por organizaciones de productores, ONG, que recogen este acervo de conocimiento y prácticas de manejo sustentables (Boege y Carranza, 2009).

Instituciones del gobierno como el INIFAP han hecho transferencia tecnológica de sistemas de rotación de cultivos y pastos en el norte del país para hacer más resilientes los sistemas productivos ganaderos a las sequías (http://www.pronacose. gob.mx/pronacose14/Contenido/Documentos/SEQUIA_Vulnerabilidad_impacto.pdf). La Conabio y Conanp trabajan para la conservación de la agrobiodiversidad, centrada en los maíces nativos a través del programa PROMAC (18 millones de pesos en el 2014; http://www.conanp.gob.mx/maiz_criollo/) además del programa de corredor biológico a lo largo del sureste de México (15 millones de dólares para 8 años del GEF-Banco Mundial). El INIFAP y la Universidad Autónoma de Chapingo poseen bancos de semilla de cultivos nativos. Institutos tecnológicos como el de Oaxaca trabajan en el rescate y la documentación de la diversidad de cultivos autóctonos como lo son el jitomate y el algodón (Ríos-Osorio, Chávez-Servia y Carrillo-Rodríguez, 2014). Estos esfuerzos ocurren alrededor de la agricultura y de sistemas productivos de agricultura campesina, principalmente en secano.

El caballo de batalla de la Secretaría de Agricultura (Sagarpa) para desarrollar sistemas agrícolas sustentables y resilientes es el Programa Mas Agro, que junto con el CYMMIT, dedicó 582 millones de pesos en el 2014 (http://www.sagarpa. gob.mx/saladeprensa/2012/Paginas/2014B301.aspx). Dicho programa tiene como objetivo llevar el paquete de semillas mejoradas (previsiblemente transgénicas), labranza de conservación y rotación de cultivos a 3 millones de hectáreas en manos de agricultores pequeños y medianos (Turrent-Fernández, Espinosa-Calderón, Cortés-Flores y Mejía-Andrade, 2014). 
Los temas de resiliencia y vulnerabilidad son fundamentales para entender los impactos del acelerado cambio del uso el suelo así como la intensificación de cultivos en su mayoría para exportación (Bonilla-Moheno, Aide y Clark, 2012). En el caso del aguacate en Michoacán, por ejemplo, de 13,000 ha en 1974 (Morales-Manilla y Cuevas, 2011) se han desmontado más de 175,000 ha solo en 2014 (Servicio de información agroalimentaria y pesquera de la Secretaría de Agricultura, Ganadería, Desarrollo Rural, Pesca y Alimentación). Este fenómeno lleva asociada la degradación de recursos naturales base, y por consiguiente la eventual pérdida de resiliencia a estreses en el mercado, sanitarios y climáticos, que podrán suceder en estos sistemas agrícolas comerciales. Finalmente y puesto que esta expansión también altera o sustituye sistemas tradicionales de subsistencia, está reduciendo la seguridad alimentaria de poblaciones rurales aumentando la desnutrición infantil crónica que se traduce en obesidad y síndrome metabólico en adultos de poblaciones campesinas (Gurri, 2015).

El estudio de la vulnerabilidad ha tenido múltiples consecuencias prácticas de gran relevancia para el país. Los mapas de riesgo han sido elaborados por Cenapred $(2006,2009$, 2014a, b). Se han generado también índices de vulnerabilidad climática de las ciudades mexicanas (Imco, 2012), del sector primario para el caso de Bahías de Bandera, México (Ramírez-Castillo y Meza-Ramos, 2012) así como la «Guía básica para la elaboración de atlas estatales y municipales de peligro y riesgos» elaborado por la Cenapred en 2006, 2009 y 2014 (Cenapred, 2006, 2009, 2014a, b). Se han generado índices de vulnerabilidad social y su distribución espacial para todas las entidades federativas de México (Vergara-González, 2011), así como el Índice de vulnerabilidad social municipal, México (Cenapred, 2006). Se están generando índices de capacidad adaptativa en la Cenapred en colaboración con la Conagua, el Instituto Mexicano para la Competitividad, A. C. (IMCO), y el Centro Regional de Investigaciones Multidisciplinarias (CRIM-UNAM). La Ley General del Cambio Climático contempla una serie de programas y actividades abocados tanto para la mitigación del cambio climático como a la reducción de la vulnerabilidad de áreas en riesgo así como a incrementar la resiliencia en sistemas agrícolas y productivos del país (http://www.diputados. gob.mx/LeyesBiblio/pdf/LGCC_130515.pdf).

\section{Vacíos del conocimiento, retos y perspectivas}

El análisis de los sistemas socioecológicos implica poder reconocer los desafíos ambiente-sociedad que suceden en múltiples escalas espaciotemporales. Para que exista un cambio cualitativo que permita el análisis de estos sistemas complejos primero que nada hay que reconocer que existen retos; específicamente, los que están asociados a supuestos como: la estabilidad de los sistemas, la certidumbre científica y la existencia-a priori-de expertos tomadores de decisiones. Por otro lado, las instituciones gubernamentales centralizadas, por ejemplo, también tienen serias limitaciones para responder transformando rápidamente a los sistemas socioecológicos y para poder lidiar con la incertidumbre (Armitage et al., 2009).
El uso de índices a nivel municipal es útil para planear apoyos y generar estrategias de rescate, sin embargo, ignora la variabilidad local e individual (López-Ridaura et al., 2002). Además, debido a que trabajan con variables agregadas, se tratan de manera similar variables incompatibles haciéndolos difíciles de interpretar.

Los conceptos de resiliencia, vulnerabilidad, y la sustentabilidad se han ido integrando más allá de la investigación e incorporando en el monitoreo de la dinámica de los sistemas socioecológicos, pero con alcances limitados. Los fondos de financiamiento, sin embargo, priorizan las iniciativas de corto plazo, lo cual contradice el sentido de la sustentabilidad y el poder entender procesos que impactan la vulnerabilidad y la resiliencia en los socioecosistemas. Por lo mismo, tampoco son prioritarios los esfuerzos dirigidos a desarrollar herramientas pedagógicas, y de sistematización y metodológicas. Se dan también contradicciones en las políticas y programas gubernamentales. Así, algunos programas enfatizan, por ejemplo, el rescate y el consumo de productos de la agrobiodiversidad, como los maíces nativos, mientras que otros buscan su reemplazo por variedades mejoradas.

Solo estudios integrales y de largo plazo nos permitirán observar un proceso tan elusivo como el de la resiliencia. Sin embargo, los estudios de caso son caros, toman mucho tiempo y al estar geográficamente restringidos son difíciles de extrapolar. Esto limita la aplicación de los resultados de los estudios de caso al diseño e implementación de políticas que reduzcan la vulnerabilidad (Engle, 2011).

\section{Conclusiones}

En síntesis, en México se han logrado avances importantes en el tema de sistemas socioecológicos, resiliencia y vulnerabilidad. El desarrollar sistemas socioecológicos más resilientes, menos vulnerables, que puedan ser funcionales mediante su continua adaptación y transformación mientras están expuestos a múltiples vaivenes coyunturales y estructurales, conlleva una continua retroalimentación de tipo no lineal. Para lograrlo es fundamental fortalecer no solo las interacciones interdisciplinarias entre investigadores de distintas disciplinas, sino trascender las barreras del ámbito científico para desarrollar propuestas transdisciplinarias en colaboración con diversos actores de la sociedad (Balvanera et al., 2017). Como resultado de procesos de cogeneración de conocimiento y cogeneración de alternativas surgirán arreglos de gobernanza a múltiples niveles, con su respectivos agentes sociales entrelazados de manera vertical y horizontal (Ostrom, 2005). Se requiere por lo tanto fortalecer la capacidad de generar procesos sociales flexibles e innovadores - lo cual es un ingrediente clave para la capacidad adaptativa. Los elementos como la confianza, la resolución de conflictos y el aprendizaje social son los cimientos clave para la gobernanza en un mundo que está continuamente cambiando y, sobre todo, que es desigual y con grupos y relaciones de poder asimétricas. 


\section{Agradecimientos}

Los autores agradecen a Atzimba López del IIES de la UNAM, a Alejandra Ahumada, de la Red Tsiri y a Omar Xavier Masera, su apoyo en la búsqueda de información bibliográfica. La síntesis plasmada en este ensayo surge de un evento coorganizado y cofinanciado por la Sociedad Científica Mexicana de Ecología y la Red Temática de Socioecosistemas y Sustentabilidad. La información relativa a los programas de formación de recursos humanos se deriva de un esfuerzo de la Red Temática de Socioecosistemas y Sustentabilidad, en colaboración con los Dres. Ireri Suazo y Gilberto Velázquez. Parte de esta investigación fue realizada gracias al Programa UNAM-DGAPA-PAPIIT IN 210015.

\section{Apéndices. Material adicional}

Se puede consultar material adicional a este artículo en su versión electrónica disponible en doi:10.1016/j.rmb.2017.10.005.

\section{Referencias}

Adger, W. N. (2006). Vulnerability. Global Environmental Change, 16, 268-281. Aguilar-Støen, M., Moe, S. R. y Camargo-Ricalde, S. L. (2009). Home gardens sustain crop diversity and improve farm resilience in Candelaria Loxicha, Oaxaca, México. Human Ecology, 37, 55-77.

Ahn, J. H. y Choi, H. I. (2013). A new flood index for use in evaluation of local flood severity: a case study of small ungauged catchments in korea. Journal of the American Water Resources Association, 49, 1-14.

Armitage, D. R., Plummer, R., Berkes, F., Arthur, R. I., Charles, A. T., DavidsonHunt, I. J., et al. (2009). Adaptive co-management for social-ecological complexity. Frontiers in Ecology and the Environment, 7, 95-102.

Arnés, E., Antonio, J., del Val, E. y Astier, M. (2013). Sustainability and climate variability in low-input peasant maize systems in the central Mexican highlands. Agriculture, Ecosystems \& Environment, 181, 195-205.

Astier, M., García-Barrios, L., Galván-Miyoshi, Y., González-Esquivel, C. E. y Masera, O. R. (2012). Assessing the sustainability of small farmer natural resource management systems. A critical analysis of the MESMIS Program. Ecology and Society, 17, 25.

Astier, M., Speelman, E. N., López-Ridaura, S., Masera, O. R. y GonzálezEsquivel, C. E. (2011). Sustainability indicators, alternative strategies and trade-offs in peasant agroecosystems: analysing 15 case studies from Latin America. International Journal of Agricultural Sustainability, 9, 409-422.

Balvanera, P., Castillo, A., Ávila, P., Caballero, K., Flores, A., Galicia, C., et al. (2011). Marcos conceptuales interdisciplinarios para el estudio de los servicios ecosistémicos en América Latina. En P. Laterra, E. Jobbágy, y J. Paruelo (Eds.), Valoración de servicios ecosistémicos. Conceptos, herramientas y aplicaciones para el ordenamiento territorial (pp. 39-78). Buenos Aires, Argentina: Ediciones INTA.

Balvanera, P. y Cotler, H. (2011). Los servicios ecosistémicos. CONABIO. Biodiversitas, 94, 1-7.

Balvanera, P., Daw, T. M., Gardner, T. A., Martín-López, B., Norström, A. V., Ifejika, C., et al. (2017). Key features for more successful place-based sustainability research on social-ecological systems: a Programme on Ecosystem Change and Society (PECS) perspective. Ecology and Society, 22, 14.

Berkes, F., Folke, C. y Colding, J. (2000). Linking social and ecological systems: management practices and social mechanisms for building resilience. Cambridge: Cambridge University Press.

Berkes, F. y Jolly, D. (2002). Adapting to climate change: social-ecological resilience in a Canadian western Arctic community. Conservation Ecology, $5,18$.

Binder, C., Hinkel, J., Bots, P. y Pahl-Wostl, C. (2013). Comparison of frameworks for analyzing social-ecological systems. Ecology and Society, 18, 26.
Boege, E. y Carranza, T. (2009). Agricultura sostenible campesinoindígena, soberanía alimentaria y equidad de género. Seis experiencias de organizaciones indígenas y campesinas en México. México D.F: PIDAASSA, Brot für die Welt, Xilotl Servicios Comunitarios.

Bonilla-Moheno, M., Aide, T. M. y Clark, M. L. (2012). El efecto del cambio poblacional en el uso del suelo en paisajes rurales de México: un análisis a nivel estatal. Investigación Ambiental Ciencia y Política Pública, 4, 87-100.

Brondízio, E. S. y Moran, E. F. (2012). Human-environment interactions: current and future directions. Amsterdam: Springer Science \& Business Media.

Burton, I. y Lim, B. (2001). An adaptation policy framework: capacity building for stage ii adaptation. New Yotk: UNDP-GEF, National Comumnication Support Programme.

Caballero, J., Toledo, V., Argueta, A., Aguirre, E., Rojas, P. y Viccon, J. (1978). Estudio botánico y ecológico de la región del río Uxpanapa, Veracruz, Núm. 8. Flora útil o el uso tradicional de las plantas. Biótica, 3, 103-144.

Castillo, A. (2011). Comunicación e interacciones entre las ciencias ambientales (socioecológicas) y distintos sectores de la sociedad. En A. ArguetaVillamar, E. Corona, y P. Hersch (Eds.), Saberes colectivos y diálogos de saberes en México. Cuernavaca, Morelos: Centro Regional de Investigaciones Multidisciplinarias, UNAM-Universidad Iberoamericana de Puebla.

Cenapred (Centro Nacional de Prevención de Desastres). (2009). Guía básica para la elaboración de atlas estatales y municipales de peligros y riesgos. Ciudad de México: SEGOB.

Cenapred (Centro Nacional de Prevención de Desastres). (2009). Impacto socioeconómico de los principales desastres ocurridos en la República Mexicana en el año 2007. Ciudad de México: SEGOB.

Cenapred (Centro Nacional de Prevención de Desastres). (2014a). Guía básica para la elaboración de atlas estatales y municipales de peligros y riesgos. Evaluación de la vulnerabilidad física y social. Ciudad de México: SEGOB.

Cenapred (Centro Nacional de Prevención de Desastres). (2014b). Guía básica para la elaboración de atlas estatales y municipales de peligros y riesgos. Fenómenos hidrometeorológicos. Ciudad de México: SEGOB.

Challenger, A. (2016). La aplicación del enfoque ecosistémico en la política ecológica: bases conceptuales para una gestión ambiental fundamentada en el manejo sustentable de socio-ecosistemas en México (Tesis de Doctorado en Ciencias). México D.F.: Instituto de Investigaciones en Ecosistemas y Sustentabilidad, Universidad Nacional Autónoma de México.

Challenger, A., Bocco, G., Equihua, M., Chavero, E. L. y Maass, M. (2014). La aplicación del concepto del sistema socio-ecológico: alcances, posibilidades y limitaciones en la gestión ambiental de México. Investigación Ambiental, Ciencia y Política Pública, 6, 1-21.

Chan, K. M., Guerry, A. D., Balvanera, P., Klain, S., Satterfield, T. y Basurto, X. (2012). Where are cultural and social in ecosystem services? A framework for constructive engagement. BioScience, 62, 744-756.

Conapo (Consejo Nacional de Población). (2013). Índice de marginación por entidad federativa y municipio 2010. México D.F: Consejo Nacional de Población.

Conde, C., Ferrer, R. y Orozco, S. (2006). Climate change and climate variability impacts on rainfed agricultural activities and possible adaptation measures. A Mexican case study. Atmósfera, 19, 181-194.

Daily, G. (1997). Nature's services: societal dependence on natural ecosystems. Washington D.C.: Island Press.

Daltabuit, M. C., Licón, L., Lozano, L. y Ricco, A. (1988). Ecología humana en una comunidad de Morelos. México, D.F.: Universidad Nacional Autónoma de México.

Durand-Smith, L. (2012). La naturaleza en contexto: hacia una ecología política mexicana. México D.F.: UNAM, CEIICH, CRIM, El Colegio de San Luis A.C.

Durand-Smith, L., Figueroa-Díaz, F., Chávez, G. y Genet, M. (2011). La ecología política en México ¿Dónde estamos y para dónde vamos? Estudios Sociales (Hermosillo, Son.), 19, 281-307.

Eakin, H. y Luers, A. L. (2006). Assessing the vulnerability of socialenvironmental systems. Annual Review of Environment and Resources, 31, 365.

Engle, N. L. (2011). Adaptive capacity and its assessment. Global Environmental Change, 21, 647-756. 
Fischer, J., Gardner, T. A., Bennett, E. M., Balvanera, P., Biggs, R., Carpenter, S., et al. (2015). Advancing sustainability through mainstreaming a social-ecological systems perspective. Current Opinion in Environmental Sustainability, 14, 144-149

Galán, C., Balvanera, P. y Castellarini, F. (2014). Políticas públicas hacia la sustentabilidad: integrando la visión ecosistémica. México D.F.: Conabio.

Gallopín, G. C. (2006). Linkages between vulnerability, resilience, and adaptive capacity. Global Environmental Change, 16, 293-303.

García, R. (2006). Sistemas complejos. Barcelona: Gedisa.

García-Acosta, V. (2008). Historia y desastres en América Latina. México D.F.: Red de Estudios Sociales de Prevención de Desastres en América Latina (LA RED). CIESAS.

García-Barrios, L., Speelman, E. y Pimm, M. (2008). An educational simulation tool for negotiating sustainable natural resource management strategies among stakeholders with conflicting interests. Ecological Modelling, 210, $115-126$.

Gavito, M. E., Martínez-Yrizar, A., Ahedo, R., Araiza, S., Ayala, B., Ayala, R., et al. (2014). La vulnerabilidad del socio-ecosistema de bosque tropical seco de Chamela, Jalisco, al cambio global: un análisis de sus componentes ecológicos y sociales. Investigación Ambiental, Ciencia y Política Pública, 6, 109-126.

Gurri, F. D. (2015). The disruption of subsistence agricultural systems in rural Yucatán, Mexico may have contributed to the coexistence of stunting in children with adult overweight and obesity. Collegium Antropologicum, 39, 847-854.

Imco (Instituto Mexicano para la Competitividad). (2012). Índice de vulnerabilidad climática. México D.F.: Instituto Mexicano para la Competitividad (IMCO).

Jardel, P., Maass, M. y Rivera-Monroy, V. (2013). La investigación ecológica de largo plazo en México. México D.F.: Editorial Universitaria-Universidad de Guadalajara.

Liu, J., Dietz, T., Carpenter, S. R., Folke, C., Alberti, M., Redman, C. L., et al (2007). Coupled human and natural systems. AMBIO: A Journal of the Human Environment, 36, 639-749.

López-Marrero, T. (2010). An integrative approach to study and promote natural hazards adaptive capacity: a case study of two flood-prone communities in Puerto Rico. The Geographical Journal, 176, 150-163.

López-Ridaura, S., Masera, O. y Astier, M. (2002). Evaluating the sustainability of complex socio-environmental systems. The MESMIS framework Ecological Indicators, 2, 135-148.

MA (Millenium Ecosystem Assessment). (2005). Millenium Ecosystem Assess ment. Current state and trends. Washington, D.C.: New Island.

Maass, J. M. (2012). El manejo sustentable de socio-ecosistemas. En J. L. Calva (Ed.), Cambio climático y políticas de desarrollo sustentable. Análisis estratégico para el desarrollo (pp. 267-290). México D.F.: Juan Pablos Editor, Consejo Nacional de Universitarios.

Maass, J. M., Balvanera, P., Castillo, A., Daily, G. C., Mooney, H. A., Ehrlich, P., et al. (2005). Ecosystem services of tropical dry forests: insights from longterm ecological and social research on the Pacific Coast of Mexico. Ecology and Society: A Journal of Integrative Science for Resilience and Sustainability, 10, 1-23.

Maass, J. M., Díaz-Delgado, R., Balvanera, P., Castillo, A. y Martínez-Yrízar, A. (2010). Redes de investigación ecológica y socio-ecológica a largo plazo (LTER y LTSER) en Iberoamérica: los casos de México y España. Revista Chilena de Historia Natural, 83, 171-184.

Maass, J. M. y Equihua, M. (2015). Earth stewardship, socioecosystems, the need for a transdisciplinary approach and the role of the International Long Term Ecological Research Network (ILTER). Earth Stewardship, 217-233.

Maass, J. M., Martínez-Yrízar, A., Patiño, C. y Sarukhán, J. (2002). Distribution and annual net accumulation of above-ground dead phytomass and its influence on throughfall quality in a Mexican tropical deciduous forest ecosystem. Journal of Tropical Ecology, 18, 821-834.

Masera, O., Astier, M. y López-Ridaura, S. (1999). Sustentabilidad y manejo de recursos naturales: el marco de evaluación MESMIS. Ciudad de México: Mundi-Prensa, GIRA, Instituto de Ecología.

Messner, F. y Meyer, V. (2006). Flood damage, vulnerability and risk perceptionchallenges for flood damage research. En J. Schanze, E. Zeman, y J. Marsalek
(Eds.), Flood risk management: hazards, vulnerability and mitigation measures. Amsterdam: Springer.

Monterroso-Rivas, A. I., Conde-Álvarez, C., Rosales-Dorantes, G., GómezDíaz, J. D. y Gay-García, C. (2011). Assessing current and potential rainfed maize suitability under climate change scenarios in México. Atmósfera, 24, 53-77.

Morales-Manilla, L. y Cuevas, G. (2011). Inventarios 1974-2007 y evaluación de impacto ambiental regional del cultivo del aguacate en el estado de Michoacán. Informe final. Morelia, Mich: Centro de Investigaciones en Geografía Ambiental, UNAM.

Nelson, D., Adger, W. y Brown, K. (2007). Resilience and adaptation to climate change: linkages and a new agenda. Annual Review of Environment and Resources, 32, 395-419.

O’Brien, K., Sygna, L. y Haugen, J. E. (2004). Vulnerable or resilient? A multiscale assessment of climate impacts and vulnerability in Norway. Climatic Change, 64, 193-225.

Ostrom, E. (2005). Self-governance and forest resources. Terracotta reader: a market approach to the environment. Nueva Delhi: Academic Foundation.

Palerm, Á. (1989). Antropología y marxismo. México D.F.: Editorial Nueva Imagen.

Palomo, I., Felipe-Lucía, M. R., Bennett, E. M., Martín-López, B. y Pascual, U. (2016). Disentangling the pathways and effects of ecosystem service co-production. Advances in Ecological Research, 54, 245-283.

Ramírez-Castillo, R. J. y Meza-Ramos, E. (2012). Evaluación de la vulnerabilidad del sector primario regional ante el impacto de la variabilidad climática en bahía de Banderas. México: EUMED [3 Nov 2015]. Disponible en: http://www.eumed.net

Ríos-Osorio, O., Chávez-Servia, J. L. y Carrillo-Rodríguez, J. C. (2014). Producción tradicional y diversidad de tomate (Solanum lycopersicum L.) nativo: un estudio de caso en Tehuantepec-Juchitán, México. Agricultura Sociedad y Desarrollo, 11, 35-51.

Rogé, P. y Astier, M. (2015). Changes in climate, crops, and tradition: cajete maize and the rainfed farming systems of Oaxaca, Mexico. Human Ecology, 43, 639-753.

Sarukhán, J. y Maass, J. M. (1990). Bases ecológicas para un manejo sostenido de los ecosistemas: el sistema de cuencas hidrológicas. Medio Ambiente y Desarrollo en México, 1, 81-114.

Sarukhán, J. y Soberón, J. (2008). Capital natural de México. México D.F.: Comisión Nacional para el Conocimiento y Uso de la Biodiversidad.

Smit, B. y Pilifosova, O. (2001). Adaptation to climate change in the context of sustainable development and equity. En J. J. McCarthy, O. F. Canziani, N. A. Leary, D. J. Dokken, y K. S. White (Eds.), Climate change 2001: impacts, adaptation, and vulnerability (pp. 876-912). New York: Intergovernmental Panel on Climate Change, Cambridge University Press.

Soares, D., Murillo, D., Romero, R. y Millán, G. (2014). Amenazas y vulnerabilidades: las dos caras de los desastres en Celestún. Yucatán. Desacatos, 159-177.

Torres-Rojo, J. M. y Sanginés, A. G. (2002). El potencial de México para la producción de servicios ambientales: captura de carbono y desempeño hidráulico. Gaceta Ecológica, 40-59.

Tucker, C. M., Eakin, H. y Castellanos, E. J. (2010). Perceptions of risk and adaptation: coffee producers, market shocks, and extreme weather in Central America and Mexico. Global Environmental Change, 20, 23-32.

Turrent-Fernández, A., Espinosa-Calderón, A., Cortés-Flores, J. I. y MejíaAndrade, H. (2014). Análisis de la estrategia MasAgro-maíz. Revista Mexicana de Ciencias Agrícolas, 5, 1531-1547.

Vallejo-Nieto, M. I., Gurri-García, F. D. y Molina-Rosales, D. O. (2011). Agricultura comercial, tradicional y vulnerabilidad en campesinos. Política y Cultura, 71-98.

Vergara-González, R. (2011). Vulnerabilidad social y su distribución espacial: el caso de las entidades federativas de México, 1990-2010. Paradigma Económico, 3, 85-111.

Vergara-Tenorio, M., Ellis, E. A., Aguilar, C., Antonio, J., Alarcón-Sánchez, L. D. C. y Galván-del Moral, U. (2011). La conceptualización de las inundaciones y la percepción del riesgo ambiental. Política y Cultura, $45-79$.

Wong, K. K. y Zhao, X. (2001). Living with floods: victims' perceptions in Beijiang, Guangdong, China. Area, 33, 190-201. 